\title{
Thermal and nonthermal melting of III-V compound semiconductors
}

\author{
Nikita Medvedev, ${ }^{1,2, *}$ Zhaoji Fang, ${ }^{3}$ Chenyi Xia, ${ }^{4}$ and Zheng $\mathrm{Li}^{5,6, \dagger}$ \\ ${ }^{1}$ Institute of Plasma Physics CAS, v.v.i., Za Slovankou 3, 18200 Prague, Czech Republic \\ ${ }^{2}$ Institute of Physics CAS, v.v.i., Na Slovance 2, 18221 Prague, Czech Republic \\ ${ }^{3}$ School of Electronic and Information Engineering, Beihang University, Beijing 100191, China \\ ${ }^{4}$ School of Automation Science and Electrical Engineering, Beihang University, Beijing 100191, China \\ ${ }^{5}$ Center for Free Electron Laser Science at DESY, Notkestrasse 85, D-22603 Hamburg, Germany \\ ${ }^{6}$ Max Planck Institute for the Structure and Dynamics of Matter, Luruper Chaussee 147, 22761 Hamburg, Germany
}

(Received 2 January 2019; published 10 April 2019)

\begin{abstract}
We study theoretically the response of group III-V compound semiconductors (AlAs, AlP, GaAs, GaP, GaSb) to free-electron laser irradiation, identifying their damage thresholds. The employed hybrid code XTANT is capable of modeling both thermal and nonthermal effects under ultrafast electronic excitation. It allowed us to reveal common trends in the studied materials: all but the AlAs III-V compounds studied here exhibit a phase transition into a metallic disordered state of lower density than the solid phase via a thermal phase transition. This transition is instigated by electron-ion coupling at doses below the nonthermal melting. Irradiated AlAs showed two possible phases produced: low-density and high-density liquid. We demonstrate that the transferrable tightbinding method within the Born-Oppenheimer approximation significantly overestimates the damage threshold predicting only nonthermal melting in comparison to a non-Born-Oppenheimer scheme, which accounts for both effects and their interplay.
\end{abstract}

DOI: 10.1103/PhysRevB.99.144101

\section{INTRODUCTION}

The recent advent of short-wavelength free-electron lasers (FELs) has enabled systematic and comprehensive studies of changes induced in materials by irradiation with ultrashort pulses in the photon-energy range from extreme ultraviolet $(\sim 30 \mathrm{eV})$ to hard $\mathrm{x}$ ray (up to few tens of $\mathrm{keV}$ ) [1,2]. X-ray radiation is absorbed in matter mostly via an atomic photoeffect, exciting electrons from core atomic shells into highenergy states within the conduction band $[3,4]$. The created core holes decay predominantly via the Auger process $[5,6]$, promoting secondary electrons into the conduction band on a few-femtosecond timescales, where they undergo secondary cascades alongside with the photoelectrons. Secondary electron cascades consist of a series of elastic and inelastic scattering events [7-9]. The former exchanges only kinetic energy with the target atoms or collective modes (phonons), without excitation of electrons, whereas the latter, known as impact ionization, creates new free electrons.

The cascades typically last from a few to a few-hundred femtoseconds, depending on the photon energy and the material [7], until the energy of the cascading electron drops below a certain threshold. Low-energy electrons contribute to the quasielastic electron-ion coupling, transferring kinetic energy to the lattice atoms, and can modify interatomic potential due to change in the potential energy [10]. The first channel can lead to atomic heating sufficient to induce thermal melting, typically taking place at a few-picosecond timescales

\footnotetext{
*Corresponding author: nikita.medvedev@ @zu.cz

†Corresponding author: zheng.li@cfel.de
}

[11]. The second one can induce a phase transition without sufficient heating of the atoms merely by altering the atomic bonds from attractive to repulsive ones-a process known as nonthermal melting (or, formerly, cold melting) [12].

It has been demonstrated that a complex interplay between the two melting channels in silicon can lead to unusual kinetic pathways of material damage in FEL-irradiated silicon $[12,13]$. In particular, it lowered the damage threshold with respect to the results of models that do not consider such interplay of both effects, showing their nonadditivity. In this work, we analyze the influence of the two damage mechanisms in group III-V semiconductors irradiated with an FEL pulse.

\section{MODEL}

In order to model behavior of semiconducting materials under a femtosecond FEL irradiation, the recently developed hybrid code XTANT was applied. All the details of XTANT can be found in a comprehensive review in Ref. [14]; here we only briefly describe its details. The code combines a few theoretical schemes to describe important aspects of $\mathrm{x}$-ray radiation effects in matter:

(i) FEL irradiation, photon absorption, and excitation of electrons, as well as their secondary cascading, are described within an event-by-event Monte Carlo (MC) scheme. For inelastic electron scattering (impact ionization), we used the so-called binary encounter Bethe (BEB) atomic cross sections [15], analogous to the XCASCADE code [7]; for the elastic ones, we applied the Mott cross section with the modified Molier screening parameter [8]. The calculated inelastic mean free paths for the materials of interest are shown in Appendix A. 
Atomic-ionization potentials and photoabsorption cross sections for each shell of each element were extracted from the EPICS2017 database (former EADL and EPDL databases) [6]. Orbital kinetic energies, required for BEB cross sections, were also extracted from the EPICS2017 database. The electrons within the MC module are traced until their kinetic energy falls below the threshold energy of $10 \mathrm{eV}$ counted from the bottom of the conduction band. When an electron loses its energy below this threshold, it joins the low-energy bath of electrons.

(ii) The electron bath at the bottom of the conduction band and within the valence band of the material is traced within a Boltzmann kinetic approach. Due to a "bump-on-hot-tail" typical shape of the electron distribution function under FEL irradiation $[14,16]$, we assume an instantaneous thermalization; this means that low-energy electrons always obey FermiDirac distribution, however with a temperature different from the atomic one. These electrons populate the energy levels corresponding to the transient band structure of the irradiated sample. Utilizing Fermi distribution, we can reduce the kinetic equation to rate equations that include energy and particle source and sink terms responsible for energy exchange with the highly excited electrons (traced within the MC module) and with the atomic system $[13,16]$ :

$$
\begin{aligned}
N_{e}^{\mathrm{low}}(t+d t)= & N_{e}^{\mathrm{low}}(t)+N_{e}^{\mathrm{high}}(t)-N_{\mathrm{ph}}(t) \\
& -N_{\mathrm{imp}}(t)-N_{A}(t), \\
E_{e}^{\mathrm{low}}(t+d t)= & E_{e}^{\mathrm{low}}(t)+E_{e}^{\mathrm{high}}(t)+E_{\mathrm{imp}}(t) \\
& +E_{A}(t)-E_{e-i}(t) .
\end{aligned}
$$

Here, $N_{e}^{\text {low }}$ is the density of electrons within the low-energy fraction; $N_{\text {ph }}$ is the density of electrons excited to the highenergy domain by the incoming photons from the laser pulse; $N_{\text {imp }}$ are electrons excited from the low-energy fraction by the secondary electron collisions (impact ionizations), and $N_{A}$ are those excited by Auger decays of core-shell holes. For the energy balance, correspondingly, $E_{e}^{\text {low }}$ is the energy density of the low-energy fraction of electrons; $E_{e}^{\text {high }}$ is the energy brought in or out by high-energy electrons that fell into or jumped off the low-energy domain (e.g., by photoabsorption); $E_{\text {imp }}$ is the energy delivered during impact-ionization events by the high-energy electrons; $E_{A}$ is the energy delivered by Auger decays of core-shell holes that involve valence- or conduction-band electrons; and $E_{e-\text { ion }}$ is the energy transferred to (or from) ions. Knowing the density and energy density of electrons allows us to restore the Fermi-Dirac distribution at each time step of the simulation.

The electron-ion energy exchange is calculated via the Boltzmann collision integral, $I_{e-i}[13]$ :

$$
E_{e-i}(t)=\sum_{i, j} I_{e-\text { ion }}^{i, j} E_{i}
$$

where the summation runs over all the electronic orbitals, $E_{i}$, for transitions between each pair of levels [14]. The matrix elements necessary for calculations of the collision integrals are obtained from the tight-binding Hamiltonian (see below) within the dynamical coupling scheme developed in Ref. [13].
We mention here that the first attempt at application of the code to GaAs has shown that the developed scheme produced too slow electron-ion energy exchange at low irradiation doses [17]; however, in the current work, we use high irradiation doses around and above the damage threshold, in which case the scheme works reliably well. Our developed scheme is similar to the familiar Tully's surface hopping [18], with the main difference being that we do not sample electronic hops randomly but average over all possible hops and calculate an average energy flaw between electrons and ions from it $[12,13]$. Electronic populations (distribution function) are then adjusting accordingly, thereby changing the potentialenergy surface-it is in this sense that we call it an approach beyond the Born-Oppenheimer approximation.

(iii) A transient band structure, as well as the forces acting on atoms, are obtained by diagonalizing the transient Hamiltonian of the transferable tight-binding (TB) method [19], which uses the $s p^{3} s^{*}$ basis set for the chosen materials [20]. The potential-energy surface depends on the transient state of all the atoms within the simulation box and on the electron distribution function. The parameters of the hopping integrals for group III-V semiconductors were reconstructed following the original work [20]; more details on the methodology, as well as the parameters themselves, are given in Appendix B. Eigenvalues of the Hamiltonians deliver the energy levels (band structure) required for electronic populations, while eigenfunctions enable us to construct the probabilities of electronic transitions required for the electron-ion collision integral mentioned above [13].

(iv) Atomic motion is traced with the classical molecular dynamics (MD) scheme on the potential-energy surface calculated within the above-mentioned TB approximation. In addition, the energy exchange between the atoms and the low-energy electrons from the valence and the conduction band is calculated via the Boltzmann electron-ion (electronphonon) collision integral [13]. Energy transferred from (or to) electrons is then redistributed among all the atoms in the simulation box by the velocity scaling [12]. We apply the Parrinello-Rahman barostat (NPH atomic ensemble) to allow for changes in the volume and thus the density of the irradiated samples [21]. This method enables us to identify final states of the damaged solids [12].

Knowing the atomic velocities at each time step, $\overrightarrow{v_{k}}$, we can define the velocity autocorrelation function:

$$
I(\tau, \omega)=F\left\{\frac{1}{N_{\mathrm{at}}} \sum_{k=1}^{N_{\mathrm{at}}}\left\langle\overrightarrow{v_{k}}(\tau) \overrightarrow{v_{k}}(\tau+t) \exp \left(-\alpha t^{2}\right)\right\rangle\right\},
$$

where $F$ denotes the Fourier transform, $N_{\text {at }}$ is the number of atoms in the simulation box, the parameter $\alpha$ is chosen to suppress the autocorrelation function for effective selection of the vibrational modes that are present within a given time interval of $300 \mathrm{fs}$ [14], and the brackets $\langle x(t)\rangle$ denote averaging over time $t$. This allows one to study the temporal evolution of vibrational modes at various frequencies.

The developed model and code, XTANT, has been validated on examples of diamond: the damage threshold was compared to the experimental one in Ref. [22], and the timeresolved kinetics of a phase transition in Ref. [23], which also validated our calculations of the optical parameters; 

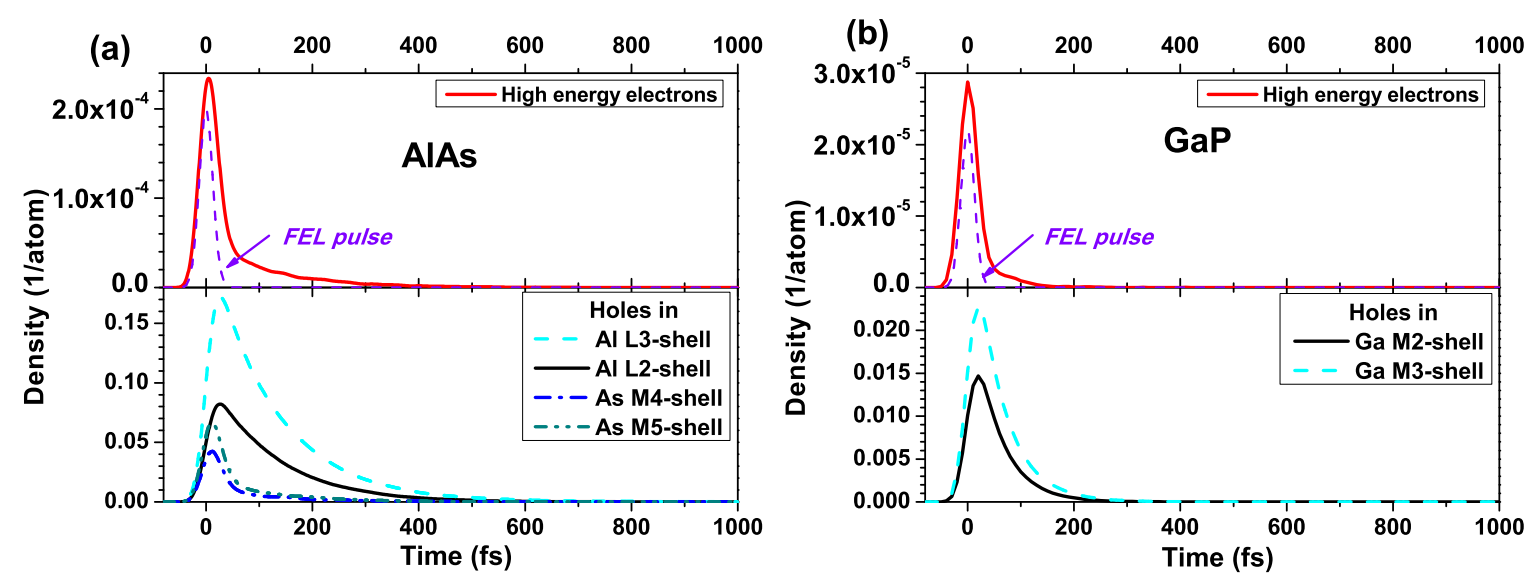

FIG. 1. (a) Densities of excited high-energy electrons (upper panel) and holes in different atomic shells (lower panel) in AlAs, irradiated with FEL of $92 \mathrm{eV}, 30 \mathrm{fs}$ FWHM, and $1.1 \mathrm{eV} /$ atom absorbed dose. (b) The same for GaP for the absorbed dose of $0.9 \mathrm{eV} /$ atom.

damage thresholds in amorphous carbon and $\mathrm{C}_{60}$ crystal were compared to experiments in Ref. [24]. The TB parameters that we use in the current manuscript were thoroughly tested for GaAs in the original papers by Molteni et al. $[20,25,26]$. Considering that parameters for other III-V compounds are constructed within the same formalism on the basis of the same data, we assume their quality to be on the same level.

\section{RESULTS}

The damage processes in all the studied compounds proceed in a similar way: they start with excitation of photoelectrons and secondary cascades. In the case of $92 \mathrm{eV}$ photon energy of the chosen FEL pulse, the cascades are very fast and finish within the pulse duration (30 fs FWHM), although Auger decay of the upper shells in some materials may take longer; see Fig. 1.

The characteristic Auger-decay times of $L_{2,3}$ shells (spinorbit splitting neglected) in $\mathrm{Al}$ atoms are $115 \mathrm{fs}$ according to Ref. [6], which allows a relatively large number of holes to be transiently accumulated there before their relaxation. Such densities of holes may also affect the interatomic potential and thereby the atomic dynamics. This effect cannot be taken into account in the present model as we only have TB hopping integrals for the valence orbitals. An effect of core holes must be analyzed within full-electron methods (especially in Al-containing materials), which is beyond the scope of the present work [27].

After high-energy electrons and core holes are relaxed, the density of low-energy conduction-band electrons reaches its peak and starts to reduce due to energy exchange with the lattice atoms. This process cools down electrons and brings some of them back to the valence band. The heating of atoms eventually leads to material disordering (melting). During melting, all III-V group materials studied here exhibited bandgap collapse, indicating metallic phases are reached in the process; see Fig. 2.

Among the studied materials, AlAs demonstrated two final phases: the low-density liquid (LDL) at lower deposited doses and the high-density liquid (HDL) at higher doses, similar to silicon [12]. A transition into HDL proceeds through the transient phase of LDL lasting for a few picoseconds. LDL is characterized by the local order present, whereas HDL is completely disordered. As the simulations run only up to 10 ps, we cannot exclude the possibility that LDL AlAs always ends up in HDL after some time, and thus LDL could be only transiently produced. In such a case, it could potentially be possible to quench this phase by a rapid cooling achievable by adjusting photon energy or an incident angle such that the established gradients of the deposited energy would allow it.

All the other studied III-V compounds only demonstrated phase transitions into disordered phases with lower densities. In these materials, the disordered phases reached by $10 \mathrm{ps}$ seem to be final, and we do not expect the phase to change at later times. Examples of atomic snapshots in Figs. 3 and 4 show that the order in irradiated $\mathrm{AlAs}$ and GaP slowly disappears, although not completely and a short-range order is still present for the doses just above the corresponding damage

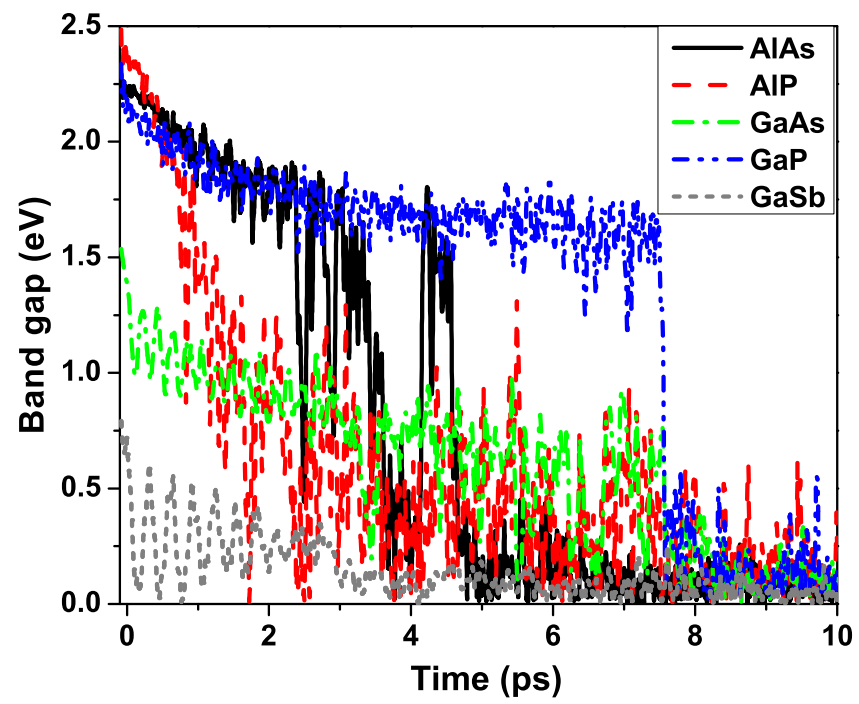

FIG. 2. Band gap in various materials irradiated with FEL pulses of $30 \mathrm{fs}$ FWHM, photon energy $92 \mathrm{eV} /$ atom. Absorbed doses were the following: in AlAs $1.1 \mathrm{eV} /$ atom, in $\mathrm{AlP} 1.7 \mathrm{eV} /$ atom, in $\mathrm{GaAs}$ $1.2 \mathrm{eV} /$ atom, in $\mathrm{GaP} 0.9 \mathrm{eV} /$ atom, and in $\mathrm{GaSb} 0.8 \mathrm{eV} /$ atom. 


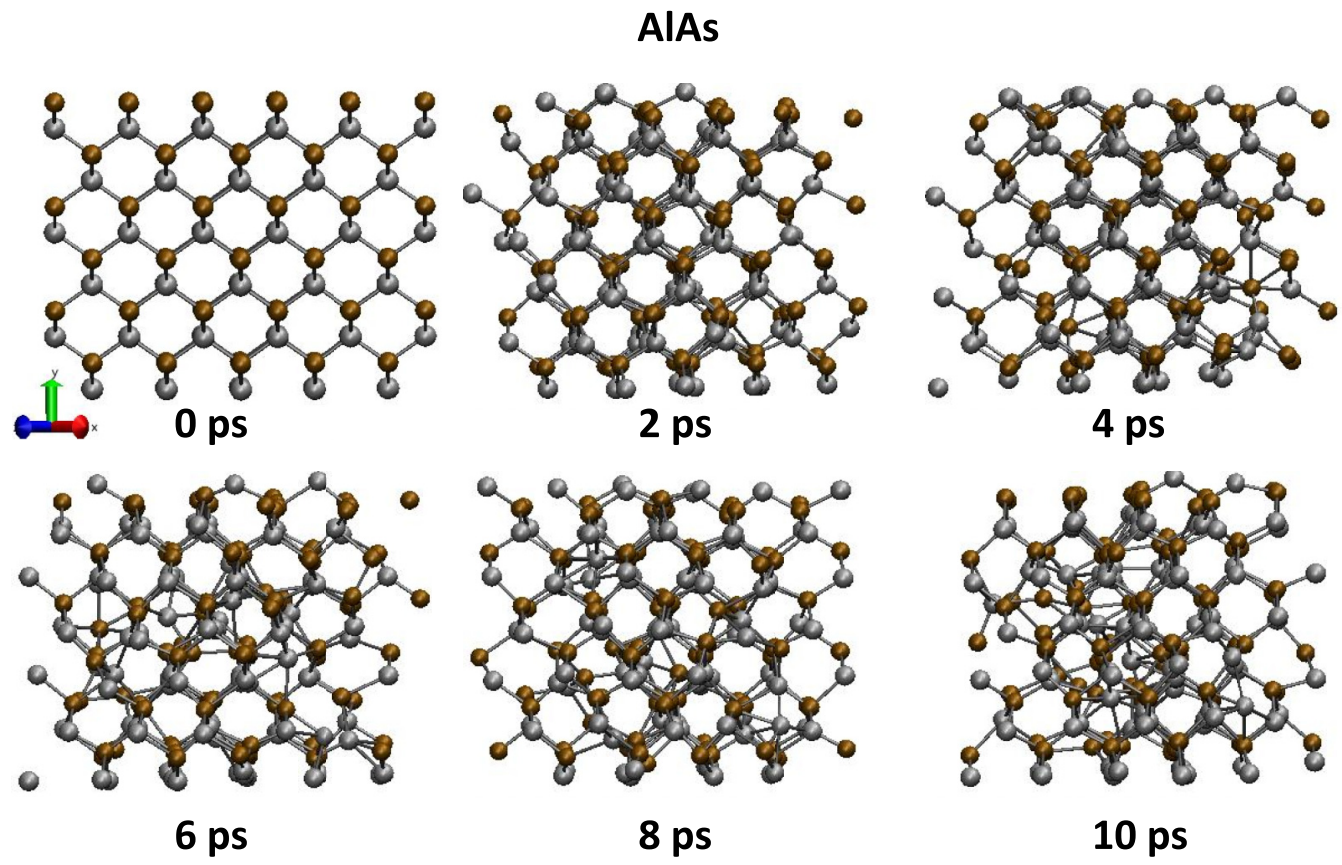

FIG. 3. Atomic snapshots of AlAs irradiated with an FEL pulse of 30 fs FWHM, photon energy $92 \mathrm{eV} /$ atom, absorbed dose of $1.1 \mathrm{eV} /$ atom. Gray balls are $\mathrm{Al}$ atoms; ocher balls are As atoms. View along $(x, z)$ diagonal, 216 atoms in the supercell.

thresholds. With increase of a dose, disorder occurs faster. The other studied materials exhibit the same behavior as GaP.

The calculated damage thresholds within the BornOppenheimer (BO) approximation and beyond (including electron-ion coupling) are summarized in Table I. We define the damage threshold as the minimal absorbed dose sufficient to trigger a macroscopic change of the material into a different phase in the bulk, without considering specific surface effects or point defect formation, consistent with our previous works, e.g., [28]. We can see that BO approximation, which excludes electron-ion energy exchange, significantly overestimates the damage thresholds - by a factor of four or more. Electron-ion coupling plays a crucial role in the damage of group III-V compounds; the same effect was also shown to take place in Si [12]. Heating of the atomic system leads to thermal damage at noticeably lower doses than a purely nonthermal melting threshold. Also, the transition of AlAs to HDL was only produced beyond the BO approximation, whereas within the BO approximation, only the LDL phase could be created.

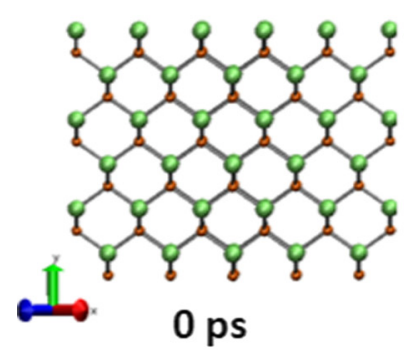
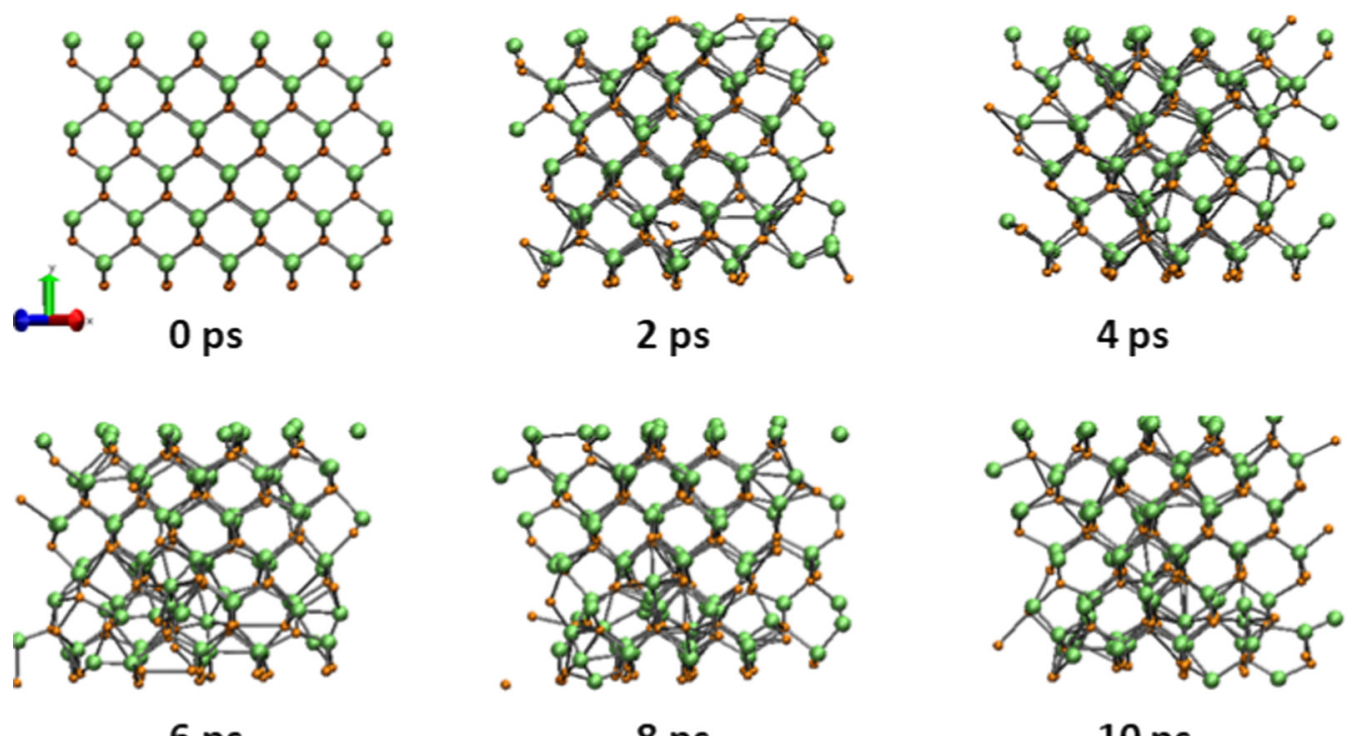

6 ps

8 ps

10 ps

FIG. 4. Atomic snapshots of GaP irradiated with an FEL pulse of $30 \mathrm{fs}$ FWHM, photon energy $92 \mathrm{eV} /$ atom, absorbed dose of $1.0 \mathrm{eV} / \mathrm{atom}$. Large green balls are Ga atoms; small orange balls are $\mathrm{P}$ atoms. View along $(x, z)$ diagonal, 216 atoms in the supercell. 
TABLE I. Calculated damage threshold doses $\left(D_{\mathrm{th}}\right)$, corresponding electron densities $\left(n_{e}\right)$, and electron temperatures $\left(T_{e}\right)$ in various materials: full model vs Born-Oppenheimer approximation (BO).

\begin{tabular}{|c|c|c|c|c|c|c|}
\hline Material & $D_{\text {th }}(\mathrm{eV} /$ atom $)$ & $n_{e}(\%)$ & $T_{e}(\mathrm{kK})$ & $D_{\text {th }}$ within BO (eV/atom) & $n_{e}$ within $\mathrm{BO}(\%)$ & $T_{e}$ within $\mathrm{BO}(\mathrm{kK})$ \\
\hline \multirow[t]{2}{*}{ AlAs } & 1.1 (LDL) & 5.5 & 11 & 8 & 27 & 40 \\
\hline & 1.2 (HDL) & 6 & 11.8 & & & \\
\hline AlP & 1.7 & 7.5 & 14 & 11 & 35 & 50 \\
\hline GaAs & 1.2 & 7 & 13 & 5.5 & 21 & 30 \\
\hline $\mathrm{GaP}$ & 0.9 & 4.8 & 11.5 & 5.9 & 22 & 30 \\
\hline $\mathrm{GaSb}$ & 0.8 & 7.5 & 9 & 3 & 18 & 20 \\
\hline $\mathrm{Si}$ & $1.0(\mathrm{LDL})$ & 5.5 & 10 & 2.7 & 15 & 18 \\
\hline
\end{tabular}

We also converted the damage threshold dose from Table I into incoming fluence, assuming the relation $F_{\text {th }}=\frac{D_{\text {th }}}{n_{\text {at }} \lambda}[1-$ $\exp (-d / \lambda)]$, where $F_{\text {th }}$ is the threshold fluence, $n_{\text {at }}$ is the atomic density of the target, $\lambda$ is the photon attenuation length taken from [29], and for the present estimation we set the energy deposition depth $d=\lambda$ [14]. The results are shown in Fig. 5 for the studied materials. Interestingly, AlP has the highest radiation resistance across the entire studied photonenergy regime: about an order of magnitude higher threshold fluence than $\mathrm{GaP}$ due to a larger photon attenuation length and a higher damage threshold dose.

We analyzed the kinetic pathways of the induced phase transitions by means of the Fourier analysis of the atomic vibrations. Utilizing the velocity autocorrelation function shown in Figs. 6 and 7 for examples of AlAs and GaP, we calculated the phonon spectra at different times after excitation, softly truncated within the 300 fs interval as mentioned in Sec. II [14]. The spectra are also shown in Figs. 6 and 7. In both shown cases, AlAs and GaP, the optical phonons at $\sim 400 \mathrm{~cm}^{-1}$ are initially strongly excited, as seen by the increased amplitude. At longer timescales of a few ps, their amplitude decreases, whereas simultaneously the amplitude of the acoustical branch at $\sim 150 \mathrm{~cm}^{-1}$ slightly increases, indicating that the energy is transferred from the optical to acoustic phonons. Some part of the kinetic energy is also transferred

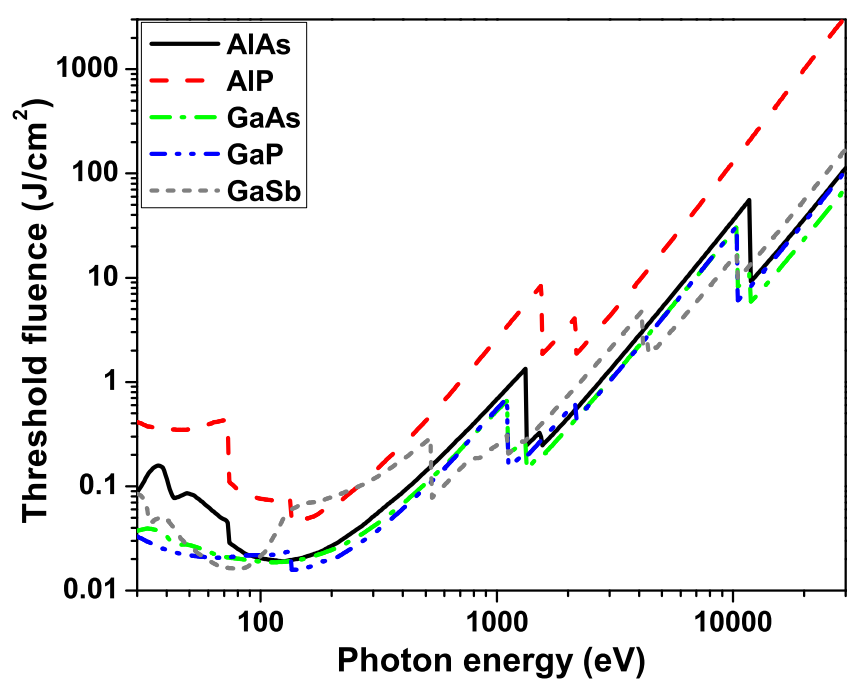

FIG. 5. Damage threshold fluence in AlAs, AlP, GaAs, GaP, and $\mathrm{GaSb}$. to the potential energy related to increasing disorder of the atomic system, which in turn reduces the amplitudes of both optical and acoustic phonons by the end of the simulations ( $\sim 10$ ps), especially in AlAs; see Fig. 6(a).

\section{DISCUSSIONS}

It remains an open question as to why, among the materials within the same group III-V compounds, only one demonstrated two liquid phases under femtosecond electronic excitation. We have compared the properties of these materials, supplementing the analysis with our previously reported diamond and silicon cases, attempting to clarify the difference. We hypothesize that the main driving factor beyond an existence of the two liquid phases is the population of $3 p$ orbitals. This conjecture stems from the fact that the bottom of the conduction band in AlAs is formed predominantly by the $3 p$ orbitals of $\mathrm{Al}$ (see Fig. 8), similarly to crystalline Si. Electrons, promoted across the band gap under an action of a laser pulse, populate those states, causing the materials to turn into an LDL phase. In contrast, other materials do not exhibit an LDL phase: diamond with $2 p$ bonds graphitizes [22,23] and $\mathrm{GaP}$ and $\mathrm{GaSb}$ with $4 p$ bonds forming the conduction band (see Fig. 8) turn into a completely disordered lowdensity phase. In AlP, the bottom of the conduction band is formed by $3 s$ orbitals, whereas $3 p$ states lie higher and thus are less populated in a laser-excited material.

Let us note that the TB parameterization based on the $s p^{3} s^{*}$ basis set from [30] seems to produce a higher damage threshold in silicon with respect to our previously used $s p^{3}$-based parameterization (see Table I): cf. the damage threshold for transition into LDL was $0.65 \mathrm{eV} /$ atom and into HDL phase was $0.9 \mathrm{eV} /$ atom in [31]. Moreover, it predicts only a low-density liquid phase for both thermal as well as nonthermal melting in silicon, whereas our previous results within the $s p^{3}$ basis set demonstrated a high-density liquid phase in the nonthermal phase transition, in agreement with experiment [12]. The same effects may be present for the studied group III-V compounds. Thus, the calculated damage threshold values and reached phases in these materials need to be validated in dedicated experiments.

Despite the fact that behavior of III-V compounds under optical or near-infrared (NIR) laser pulses was extensively studied in the literature (see, e.g., [32,33]), it is not straightforward to convert the optical damage threshold fluence into the absorbed dose for an appropriate comparison (due to nonlinear photoabsorption in the optical or NIR case). 
(a)

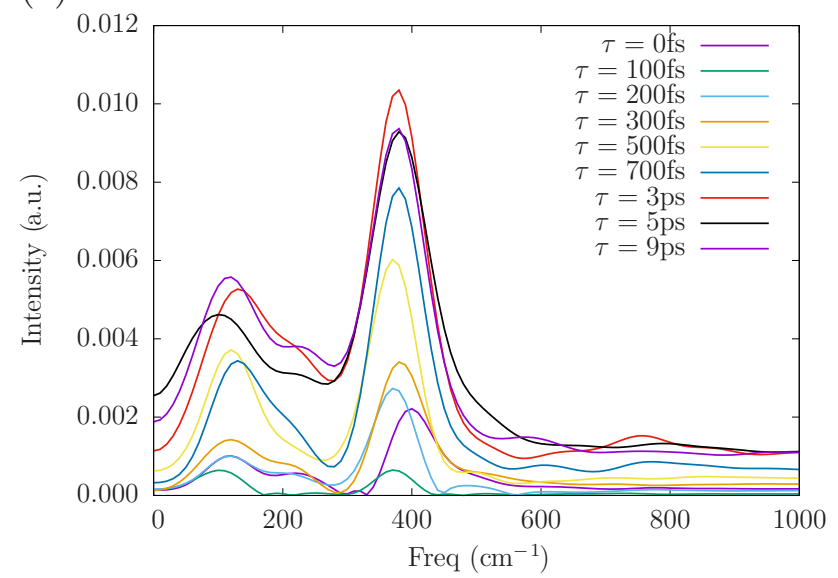

(b)

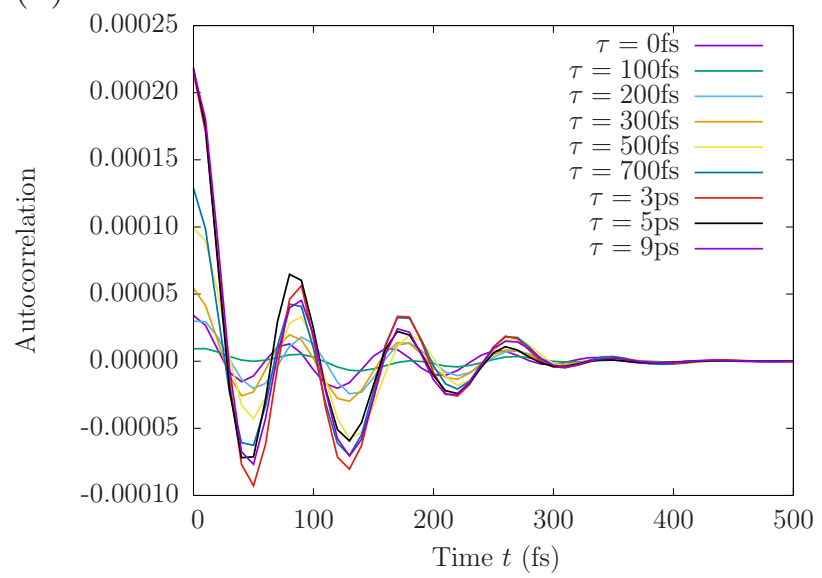

FIG. 6. (a) Vibrational spectra and (b) velocity autocorrelation functions of AlAs irradiated with an FEL pulse of 30 fs FWHM, photon energy $92 \mathrm{eV} /$ atom, absorbed dose of $1.1 \mathrm{eV} /$ atom.

However, qualitatively, the sequence of processes in irradiated materials reported in Ref. [33] matches well the observations reported here: excitation of electrons into the conduction band leads to band-gap collapse at the picoseconds timescale, which in turn triggers an irreversible atomic transition. The authors also reported that with increase of the laser fluences, the band gap collapses faster, also in agreement with the present results [33].

Last but not least, let us mention that the presented results demonstrated that the thermal melting threshold is below the nonthermal one in the group III-V compounds. These results allow one to conclude that thermal models, such as atomistic-continuum approaches (TTM-MD [34]), could be applied at deposited energies around the thermal damage as long as it is significantly below the nonthermal damage limit. This conclusion can facilitate and justify applications of such simpler models.

\section{CONCLUSIONS}

In conclusion, we introduced transferrable tight-binding parameterizations for group III-V compounds (namely, AlAs, AlP, GaAs, GaP, GaSb) into our hybrid approach XTANT and studied the behavior of these materials under FEL irradiation. All of the materials demonstrated qualitatively the same kinetics of damage into a metallic disordered phase of lower density via thermal melting (metallic liquid), except for AlAs which transitions into low-density liquid only transiently, whereas after a few picoseconds it turns into high-density liquid. The melting was induced by electron-ion coupling, which is incorporated into our approach beyond the BornOppenheimer (BO) approximation. The energy exchange took place predominantly between electrons and optical phonons, which later transferred energy into acoustical branches via phonon-phonon coupling. It is demonstrated that BO approximation systematically overestimates the damage threshold (a)

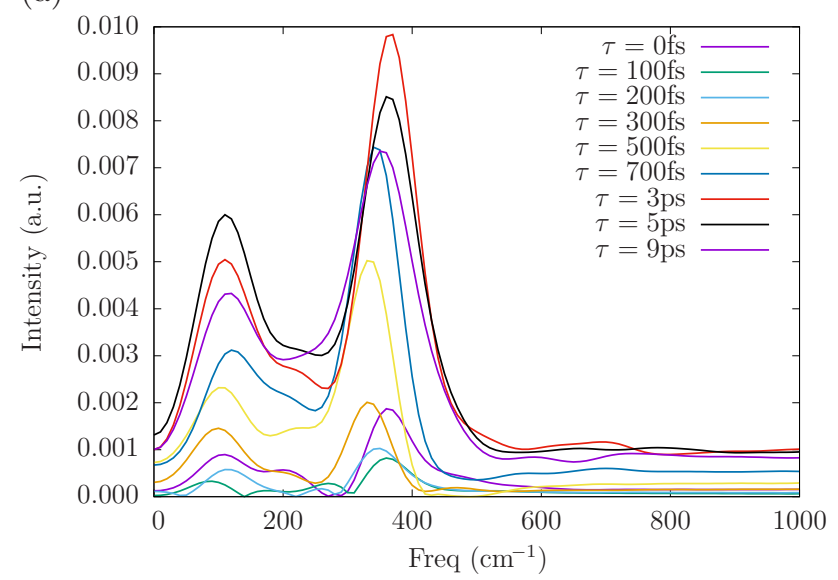

(b)

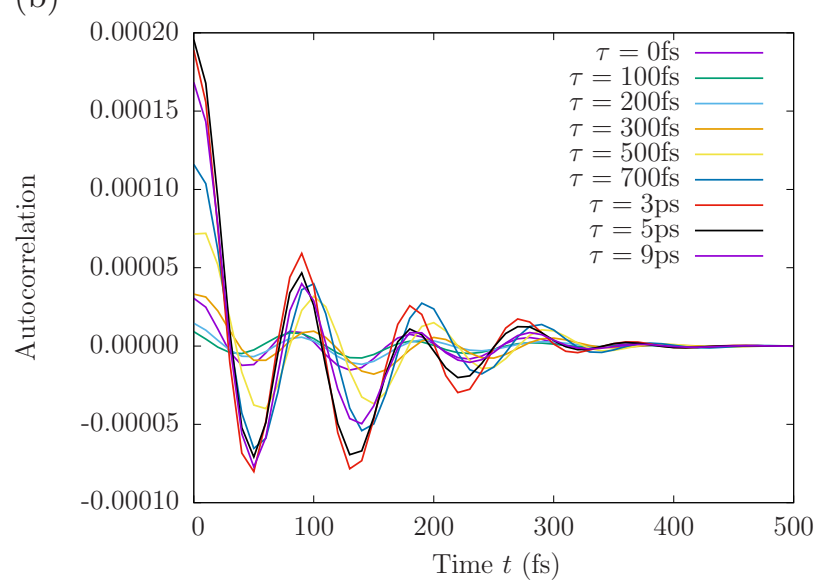

FIG. 7. (a) Vibrational spectra and (b) velocity autocorrelation functions of GaP irradiated with an FEL pulse of 30 fs FWHM, photon energy $92 \mathrm{eV} /$ atom, absorbed dose of $1.0 \mathrm{eV} /$ atom. 

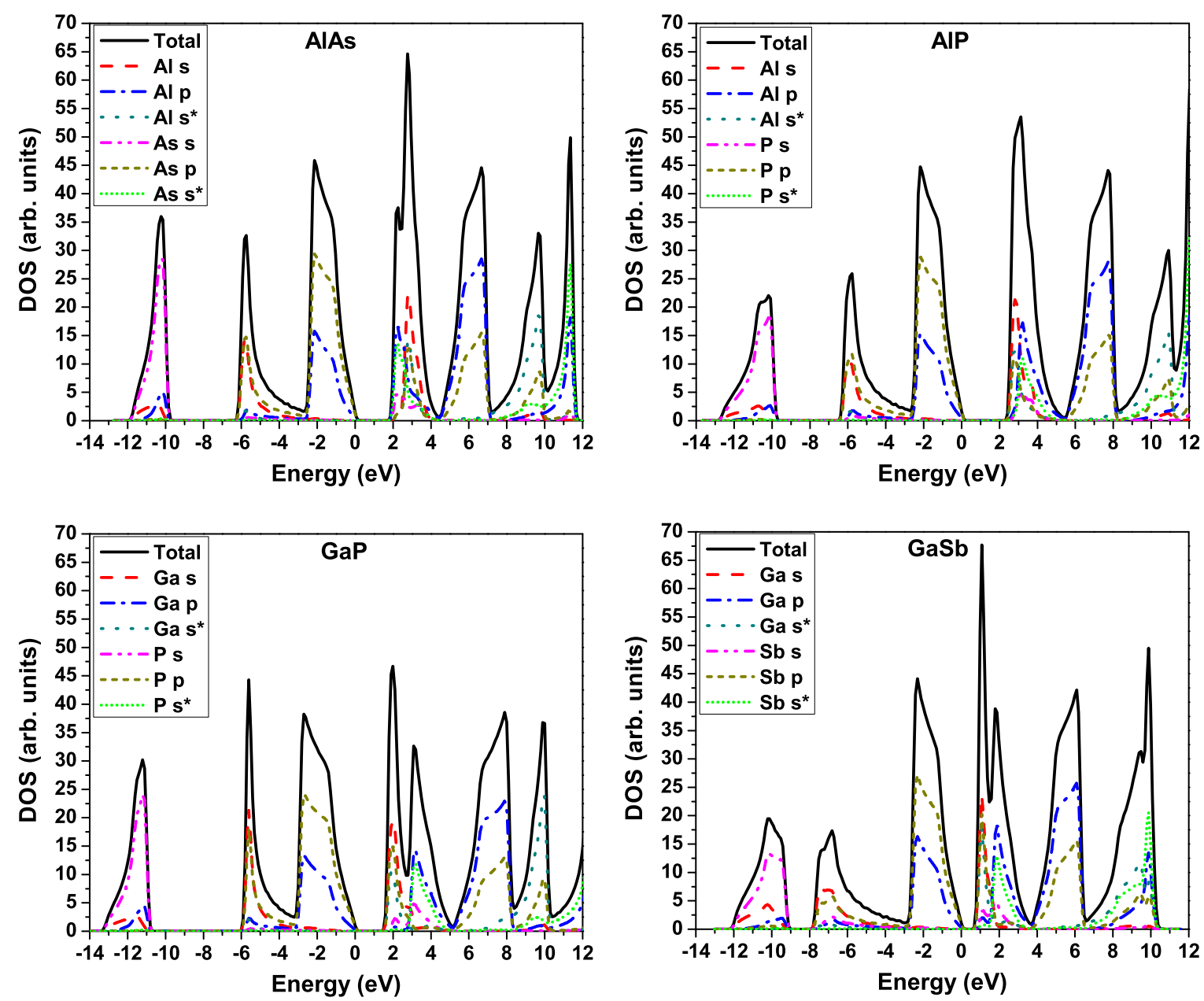

FIG. 8. Partial and total density of states in AlAs (top left), AlP (top right), GaP (bottom left), and GaSb (bottom right) calculated with our tight-binding parameters.

because it predicts only nonthermal melting. We evaluated damage thresholds in terms of the incoming fluence, absorbed dose, density of excited electrons, or peak electronic tempera-

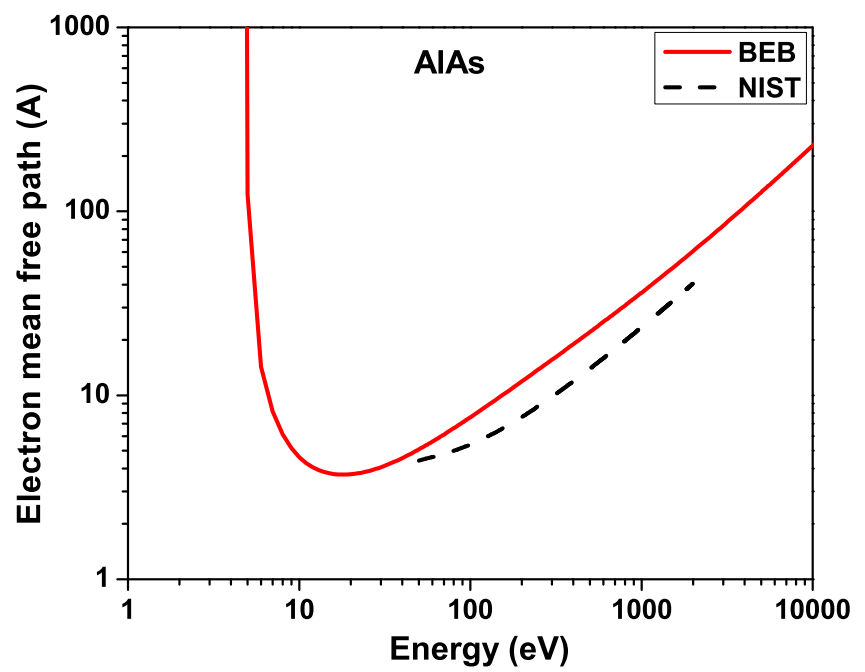

FIG. 9. Calculated BEB electron inelastic mean free path in AlAs, compared with NIST database [35]. tures, which can serve as guidance for future experiments and applications.

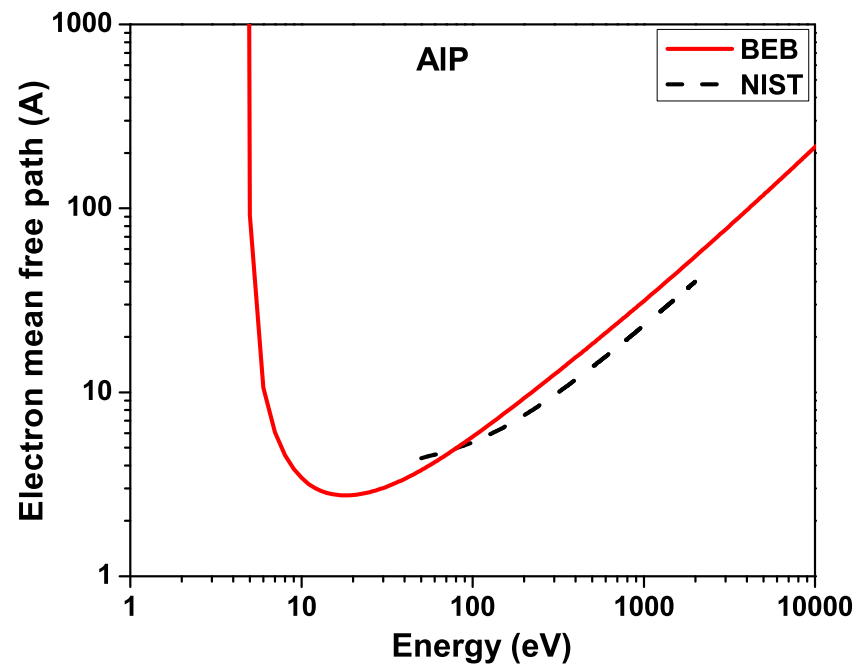

FIG. 10. Calculated BEB electron inelastic mean free path in AlAs, compared with NIST database [35]. 


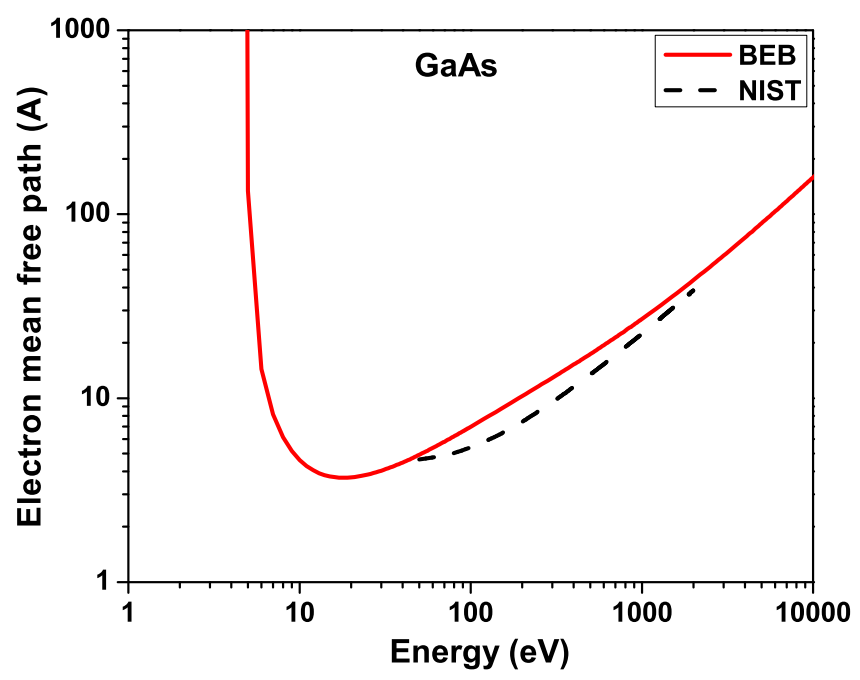

FIG. 11. Calculated BEB electron inelastic mean free path in GaAs, compared with NIST database [35].

\section{ACKNOWLEDGMENT}

Partial financial support from the Czech Ministry of Education, Youth and Sports (Grants No. LTT17015 and No. LM2015083) is acknowledged by N.M.

\section{APPENDIX A: ELECTRON MEAN FREE PATHS}

In this work, we used a simple atomic approximation for the cross sections, the so-called binary encounter Bethe (BEB) [15]. The advantage of using such a simple model is computational speedup in comparison with other more sophisticated approaches. Despite its simplicity, the produced mean free paths agree with the available data within $\sim 30 \%$ in the worst case, or typically better; see Figs. 9-13.

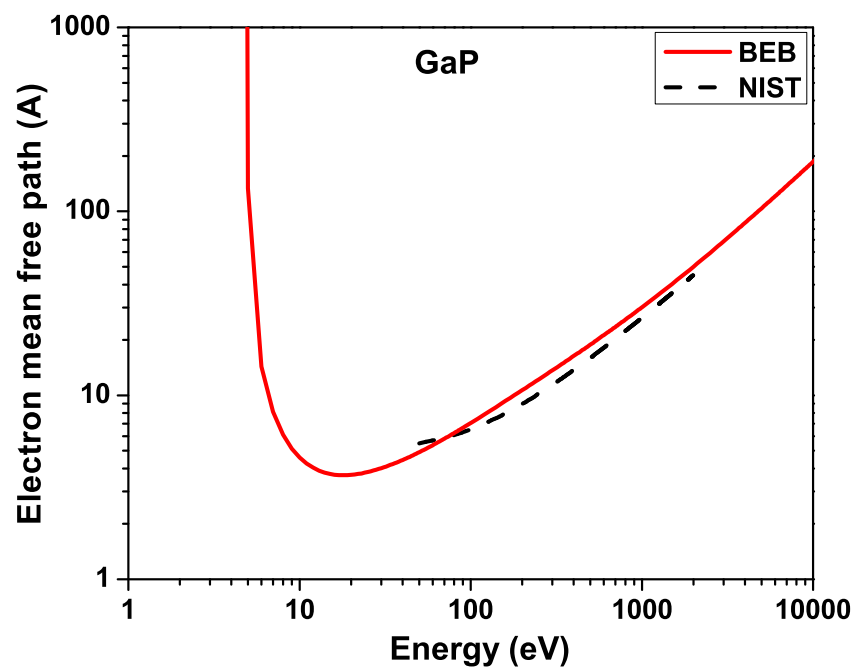

FIG. 12. Calculated BEB electron inelastic mean free path in $\mathrm{GaP}$, compared with NIST database [35].

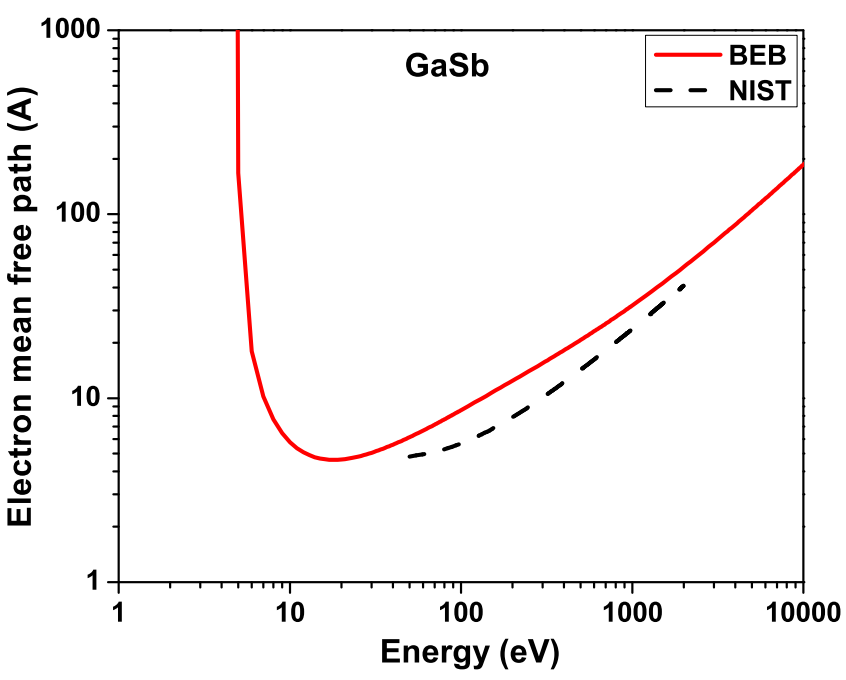

FIG. 13. Calculated BEB electron inelastic mean free path in GaSb, compared with NIST database [35].

\section{APPENDIX B: TRANSFERABLE TIGHT-BINDING PARAMETERS}

Total energy $E_{\text {tot }}$ in the transferable tight-binding approach is written in terms of two contributions, i.e., the energy from the electronic orbitals $E_{\mathrm{bs}}$ (valence- and conduction-band structure) and a repulsive core-core part $E_{\text {rep }}$ [20]:

$$
E_{\mathrm{tot}}=E_{\mathrm{bs}}+E_{\mathrm{rep}}
$$

The band structure energy $E_{\mathrm{bs}}$ is obtained as a sum over all electronic states with the current distribution function defining fractional occupation numbers, $f\left(\varepsilon_{i}\right)$ (normalized to 2 due to spin degeneracy),

$$
E_{\mathrm{bs}}=\sum_{i=1}^{N_{\mathrm{orb}}} \varepsilon_{i} f\left(\varepsilon_{i}\right),
$$

where the summation is over all the orbitals $N_{\text {orb}}$, and electronic eigenstates are obtained by diagonalization of the transient TB Hamiltonian,

$$
\varepsilon_{i}=\langle i|\hat{H}| i\rangle .
$$

TABLE II. Tight-binding overlap integrals parameters for AlAs entering Eq. (B4).

\begin{tabular}{lcccc}
\hline \hline & Al-Al & Al-As & As-Al & As-As \\
\hline$E_{s}$ & -1.163 & -1.163 & -7.527 & -7.527 \\
$E_{p}$ & 3.587 & 3.587 & 0.983 & 0.983 \\
$E_{s *}$ & 6.727 & 6.727 & 7.483 & 7.483 \\
$V_{\mathrm{ss}}$ & -2.0 & -1.666 & -1.666 & -1.420 \\
$V_{\mathrm{sp}}$ & 2.1 & 2.380 & 2.213 & 2.1 \\
$V_{p p(\sigma)}$ & 2.2 & 2.615 & 2.615 & 3.1 \\
$V_{p p(\pi)}$ & -0.67 & -0.603 & -0.603 & -0.79 \\
$V_{s * p}$ & 2.0 & 2.163 & 1.958 & 1.8 \\
$r_{0}(\AA)$ & 2.46 & 2.45 & 2.45 & 2.49 \\
\hline \hline
\end{tabular}


TABLE III. Tight-binding overlap integrals parameters for AlP entering Eq. (B4).

\begin{tabular}{lcccr}
\hline \hline & $\mathrm{Al}-\mathrm{Al}$ & $\mathrm{Al}-\mathrm{P}$ & $\mathrm{P}-\mathrm{Al}$ & \multicolumn{1}{c}{$\mathrm{P}-\mathrm{P}$} \\
\hline$E_{s}$ & -1.253 & -1.253 & -7.847 & -7.847 \\
$E_{p}$ & 4.283 & 4.283 & 1.317 & 1.317 \\
$E_{s *}$ & 7.423 & 7.423 & 8.707 & 8.707 \\
$V_{\mathrm{ss}}$ & -2.0 & -1.863 & -1.863 & -1.139 \\
$V_{\mathrm{sp}}$ & 2.1 & 2.285 & 2.271 & 1.684 \\
$V_{p p(\sigma)}$ & 2.2 & 3.013 & 3.013 & 2.486 \\
$V_{p p(\pi)}$ & -0.67 & -0.616 & -0.616 & -0.634 \\
$V_{s * p}$ & 2.0 & 2.009 & 2.274 & 1.444 \\
$r_{0}(\AA)$ & 2.46 & 2.36 & 2.36 & 2.23 \\
\hline \hline
\end{tabular}

The Hamiltonian is represented in the Koster-Slater form of overlap integrals [36], as in our previous works [16], radial parts of which are set to be functions of an interatomic distance following Harrison's rule [37]. For computational efficiency, Harrison's rule was augmented with a smooth cutoff function, quickly decaying between the second- and the third-nearest neighbors, resulting in the following expression [25]:

$$
V_{\xi}=\left(\frac{r_{0}}{r}\right)^{2} \frac{1}{1+\exp \left(\frac{2\left(r-r_{\mathrm{cut}}\right)}{d}\right)}
$$

Here, $r_{0}$ is the equilibrium bond length, $\xi$ stands for the angular momentum expansion index, which within the chosen $s p^{3} s^{*}$ basis set runs through $V_{\mathrm{ss}}, V_{\mathrm{sp}}, V_{p p(\sigma)}, V_{p p(\pi)}, V_{s * p}$, whereas all $V_{s s}^{*}$ parameters are assumed to be equal to zero [20]. On-site energies $E_{s}, E_{p}$, and $E_{s *}$ are chosen to be constants in the current approach. The smooth cutoff function added to the Harrison's rule also ensures no sharp changes in the atomic forces during molecular dynamics simulations.

The repulsive potential developed in $[20,25]$ reads

$$
\begin{aligned}
E_{\text {rep }}= & \frac{1}{2} \sum_{i \neq j}^{N_{\text {at }}}\left[\phi_{1} \exp \left(-\frac{r_{i j}-r_{0}}{\alpha}\right)+\phi_{2} \frac{r_{0}}{r_{i j}}\right] \\
& \times \frac{1}{1+\exp \left(\frac{2\left(r_{i j}-r_{\text {cut }}\right)}{d}\right)},
\end{aligned}
$$

TABLE IV. Tight-binding overlap integrals parameters for GaAs entering Eq. (B4).

\begin{tabular}{lcccc}
\hline \hline & Ga-Ga & Ga-As & As-Ga & As-As \\
\hline$E_{s}$ & -2.657 & -2.657 & -8.343 & -8.343 \\
$E_{p}$ & 3.669 & 3.669 & 1.041 & 1.041 \\
$E_{s *}$ & 6.739 & 6.739 & 8.591 & 8.591 \\
$V_{\mathrm{ss}}$ & -2.0 & -1.613 & -1.613 & -1.42 \\
$V_{\mathrm{sp}}$ & 2.1 & 2.504 & 1.940 & 2.1 \\
$V_{p p_{(\sigma)}}$ & 2.2 & 3.028 & 3.028 & 3.1 \\
$V_{p p_{(\pi)}}$ & -0.67 & -0.781 & -0.781 & -0.79 \\
$V_{s * p}$ & 2.0 & 2.082 & 2.097 & 1.8 \\
$r_{0}(\AA)$ & 2.45 & 2.45 & 2.45 & 2.45 \\
\hline \hline
\end{tabular}

TABLE V. Tight-binding overlap integrals parameters for $\mathrm{GaP}$ entering Eq. (B4).

\begin{tabular}{lcccr}
\hline \hline & Ga-Ga & Ga-P & \multicolumn{1}{c}{$\mathrm{P}-\mathrm{Ga}$} & \multicolumn{1}{c}{$\mathrm{P}-\mathrm{P}$} \\
\hline$E_{s}$ & -2.198 & -2.198 & -8.112 & -8.112 \\
$E_{p}$ & 4.115 & 4.115 & 1.125 & 1.125 \\
$E_{s *}$ & 7.185 & 7.185 & 8.515 & 8.515 \\
$V_{\mathrm{ss}}$ & -2.0 & -1.868 & -1.868 & -1.770 \\
$V_{\mathrm{sp}}$ & 2.1 & 2.736 & 1.852 & 2.618 \\
$V_{p p_{(\sigma)}}$ & 2.2 & 3.106 & 3.106 & 3.865 \\
$V_{p p(\pi)}$ & -0.67 & -0.746 & -0.746 & -0.985 \\
$V_{s * p}$ & 2.0 & 2.206 & 2.015 & 2.244 \\
$r_{0}(\AA)$ & 2.46 & 2.36 & 2.36 & 2.23 \\
\hline \hline
\end{tabular}

where the summation is running through all the pairs of atoms; $N_{\text {at }}$ is the number of atoms in the simulation box. Note that it contains the same smooth cutoff function as in Eq. (B4).

Following the methodology presented by Molteni et al. in [20], we reconstructed sets of parameters of the transferrable tight-binding model for III-V compounds, namely, AlP, AlAs, GaP, GaSb, and GaAs. The anion-cation overlap integral parameters were extracted from the paper of Vogl et al. [38], overlap integrals of the same kind of atoms were constructed from the data presented in the paper of O'Reilly et al. [39], and the parameters for the repulsive terms were taken from Molteni's et al. work [20]. The parameters given in the Cartesian form of $V(x, x)$ and $V(x, y)$ are converted into the spherical ones $V_{p p(\sigma)}$ and $V_{p p(\pi)}$ by defining the direction cosines $(l, m, n)$ of the vector connecting two atoms, and Koster-Slater representations [36]:

$$
\begin{aligned}
& V(s, x)=l V_{s p \sigma}, \\
& V(x, x)=l^{2} V_{p p \sigma}+\left(1-l^{2}\right) V_{p p \pi}, \\
& V(x, y)=\operatorname{lm}\left(V_{p p \sigma}-V_{p p \pi}\right) .
\end{aligned}
$$

Keeping in mind that all the compounds in this work have $\mathrm{ZnS}$ structure, for cation-anion interactions the direction cosines are $l=m=n=1 / \sqrt{3}$.

All the parameters for the overlap integrals and the repulsive potential are listed in Tables II-VII below; on-site energies $E_{\xi}$ and overlap integral parameters $V_{\xi}$ are in $\mathrm{eV}$.

TABLE VI. Tight-binding overlap integrals parameters for $\mathrm{GaSb}$ entering Eq. (B4).

\begin{tabular}{lcrrr}
\hline \hline & Ga-Ga & Ga-Sb & \multicolumn{1}{c}{$\mathrm{Sb}-\mathrm{Ga}$} & \multicolumn{1}{c}{$\mathrm{Sb}-\mathrm{Sb}$} \\
\hline$E_{s}$ & -3.899 & -3.899 & -7.321 & -7.321 \\
$E_{p}$ & 2.915 & 2.915 & 0.855 & 0.855 \\
$E_{s *}$ & 5.985 & 5.985 & 6.635 & 6.635 \\
$V_{\mathrm{ss}}$ & -2.0 & -1.539 & -1.539 & -1.254 \\
$V_{\mathrm{sp}}$ & 2.1 & 2.021 & 2.148 & 1.854 \\
$V_{p p}(\sigma)$ & 2.2 & 2.459 & 2.459 & 2.737 \\
$V_{p p_{(\pi)}}$ & -0.67 & -0.637 & -0.637 & -0.697 \\
$V_{s * p}$ & 2.0 & 1.826 & 2.161 & 1.589 \\
$r_{0}(\AA)$ & 2.46 & 2.64 & 2.64 & 2.65 \\
\hline \hline
\end{tabular}


TABLE VII. Repulsive potential parameters entering Eq. (B5).

\begin{tabular}{lccc}
\hline \hline Material & $\alpha$ & $\varphi_{1}(\mathrm{eV})$ & $\varphi_{2}(\mathrm{eV})$ \\
\hline $\mathrm{AlAs}$ & 0.3220 & 2.0866 & 1.1395 \\
$\mathrm{AlP}$ & 0.3088 & 2.2283 & 1.2270 \\
$\mathrm{GaAs}$ & 0.3555 & 2.3906 & 1.2347 \\
$\mathrm{GaP}$ & 0.3580 & 2.6183 & 1.2027 \\
$\mathrm{GaSb}$ & 0.3802 & 2.0136 & 1.5587 \\
\hline \hline
\end{tabular}

In all the cases, the parameter $r_{\text {cut }}=1.36 r_{0}$, and $d=0.2 \AA$ [25].

To validate the quality of the obtained TB parameters, we evaluated the equilibrium nearest-neighbor distances and the
TABLE VIII. Calculated band gaps $E_{\text {gap }}$ and nearest-neighbor distances $d_{\mathrm{NN}}$ compared to experimental values.

\begin{tabular}{lcccc}
\hline \hline Material & $E_{\text {gap }}(\mathrm{eV})$ & $E_{\text {gap }}$, expt. & $d_{\mathrm{NN}}(\AA)$ & $d_{\mathrm{NN}}$, expt. \\
\hline AlAs & 2.29 & 2.22 & 2.45 & 2.454 \\
$\mathrm{AlP}$ & 2.48 & 2.50 & 2.37 & 2.37 \\
$\mathrm{GaAs}$ & 1.54 & 1.52 & 2.45 & 2.448 \\
$\mathrm{GaP}$ & 2.34 & 2.35 & 2.37 & 2.36 \\
$\mathrm{GaSb}$ & 0.79 & 0.81 & 2.64 & 2.64 \\
\hline \hline
\end{tabular}

band gaps of the materials prior to simulation runs. The results are shown in Table VIII, demonstrating a good agreement with experimental data [40].
[1] E. L. Saldin, E. A. Schneidmiller, and M. V. Yurkov, The Physics of Free Electron Lasers (Springer, Berlin, 2000).

[2] P. Schmüser, M. Dohlus, J. Rossbach, and C. Behrens, FreeElectron Lasers in the Ultraviolet and X-Ray Regime (Springer, Cham, 2014).

[3] S. P. Hau-Riege, High-Intensity X-Rays - Interaction with Matter: Processes in Plasmas, Clusters, Molecules and Solids (Willey-VCH Verlag, Weinheim, Germany, 2011).

[4] L. Young, K. Ueda, M. Gühr, P. H. Bucksbaum, M. Simon, S. Mukamel, N. Rohringer, K. C. Prince, C. Masciovecchio, M. Meyer, A. Rudenko, D. Rolles, C. Bostedt, M. Fuchs, D. A. Reis, R. Santra, H. Kapteyn, M. Murnane, H. Ibrahim, F. Légaré, M. Vrakking, M. Isinger, D. Kroon, M. Gisselbrecht, A. L'Huillier, H. J. Wörner, and S. R. Leone, J. Phys. B At. Mol. Opt. Phys. 51, 032003 (2018).

[5] O. Keski-Rahkonen and M. O. Krause, At. Data Nucl. Data Tables 14, 139 (1974)

[6] D. E. Cullen, A Survey of Atomic Binding Energies for Use in EPICS2017 (Vienna, 2018), https://www.nndc.bnl.gov/endf/ epics/.

[7] N. Medvedev, Appl. Phys. B 118, 417 (2015).

[8] Monte Carlo Transport of Electrons and Photons, edited by T. M. Jensen, W. R. Nelson, and A. Rindi (Springer, Boston, 1988).

[9] J. Baró, J. Sempau, J. M. Fernández-Varea, and F. Salvat, Nucl. Instrum. Meth. Phys. Res. Sect. B Beam Interact. Mater. At. 100, 31 (1995).

[10] K. Sokolowski-Tinten, J. Bialkowski, M. Boing, A. Cavalleri, and D. von der Linde, Phys. Rev. B 58, R11805 (1998).

[11] B. Rethfeld, D. S. Ivanov, M. E. Garcia, and S. I. Anisimov, J. Phys. D. Appl. Phys. 50, 193001 (2017).

[12] N. Medvedev, Z. Li, and B. Ziaja, Phys. Rev. B 91, 054113 (2015)

[13] N. Medvedev, Z. Li, V. Tkachenko, and B. Ziaja, Phys. Rev. B 95, 014309 (2017).

[14] N. Medvedev, V. Tkachenko, V. Lipp, Z. Li, and B. Ziaja, 4open 1, 3 (2018).

[15] Y.-K. Kim and M. E. Rudd, Phys. Rev. A 50, 3954 (1994).

[16] N. Medvedev, H. O. Jeschke, and B. Ziaja, New J. Phys. 15, 015016 (2013).

[17] V. Tkachenko, N. Medvedev, V. Lipp, and B. Ziaja, High Energy Density Phys. 24, 15 (2017).

[18] J. C. Tully, J. Chem. Phys. 93, 1061 (1990).
[19] C. H. Xu, C. Z. Wang, C. T. Chan, and K. M. Ho, J. Phys. Condens. Matter 4, 6047 (1992).

[20] C. Molteni, L. Colombo, and L. Miglio, J. Phys. Condens. Matter 6, 5243 (1994).

[21] M. Parrinello and A. Rahman, Phys. Rev. Lett. 45, 1196 (1980).

[22] J. Gaudin, N. Medvedev, J. Chalupský, T. Burian, S. DastjaniFarahani, V. Hájková, M. Harmand, H. O. Jeschke, L. Juha, M. Jurek, D. Klinger, J. Krzywinski, R. A. Loch, S. Moeller, M. Nagasono, C. Ozkan, K. Saksl, H. Sinn, R. Sobierajski, P. Sovák, S. Toleikis, K. Tiedtke, M. Toufarová, T. Tschentscher, V. Vorlíček, L. Vyšín, H. Wabnitz, and B. Ziaja, Phys. Rev. B 88, 060101(R) (2013).

[23] F. Tavella, H. Höppner, V. Tkachenko, N. Medvedev, F. Capotondi, T. Golz, Y. Kai, M. Manfredda, E. Pedersoli, M. J. Prandolini, N. Stojanovic, T. Tanikawa, U. Teubner, S. Toleikis, and B. Ziaja, High Energy Density Phys. 24, 22 (2017).

[24] M. Toufarová, V. Hájková, J. Chalupský, T. Burian, J. Vacík, V. Vorlíček, L. Vyšín, J. Gaudin, N. Medvedev, B. Ziaja, M Nagasono, M. Yabashi, R. Sobierajski, J. Krzywinski, H. Sinn, M. Störmer, K. Koláček, K. Tiedtke, S. Toleikis, and L. Juha, Phys. Rev. B 96, 214101 (2017).

[25] C. Molteni, L. Colombo, and L. Miglio, J. Phys. Condens. Matter 6, 5255 (1994).

[26] C. Molteni, L. Colombo, and L. Miglio, Phys. Rev. B 50, 4371 (1994).

[27] Y. Rosandi, F. C. Kabeer, Y. Cherednikov, E. S. Zijlstra, M. E. Garcia, N. A. Inogamov, and H. M. Urbassek, Matererial Res. Lett. 3, 149 (2015).

[28] R. Follath, T. Koyama, V. Lipp, N. Medvedev, K. Tono, H. Ohashi, L. Patthey, M. Yabashi, and B. Ziaja, Sci. Rep. 9, 2029 (2019).

[29] B. L. Henke, E. M. Gullikson, and J. C. Davis, At. Data Nucl. Data Tables 54, 181 (1993).

[30] J. S. Graves and R. E. Allen, Phys. Rev. B 58, 13627 (1998).

[31] N. Medvedev and V. Lipp, in Proceedings of SPIE, edited by L. Juha, S. Bajt, and R. Soufli (The International Society for Optical Engineering, 2017).

[32] K. Sokolowski-Tinten, H. Schulz, J. Bialkowski, and D. Linde, Appl. Phys. A Solids Surf. 53, 227 (1991).

[33] E. N. Glezer, Y. Siegal, L. Huang, and E. Mazur, Phys. Rev. B 51, 6959 (1995).

[34] V. P. Lipp, B. Rethfeld, M. E. Garcia, and D. S. Ivanov, Phys. Rev. B 90, 245306 (2014). 
[35] C. J. Powell and A. Jablonsky, http://www.nist.gov/srd/nist71. cfm (1999).

[36] J. C. Slater and G. F. Koster, Phys. Rev. 94, 1498 (1954).

[37] W. A. Harrison, Electronic Structure and the Properties of Solids: The Physics of the Chemical Bond (Dover, New York, 2012).
[38] P. Vogl, H. P. Hjalmarson, and J. D. Dow, J. Phys. Chem. Solids 44, 365 (1983).

[39] E. P. O'Reilly and J. Robertson, Phys. Rev. B 34, 8684 (1986).

[40] A collection of III-V compounds data is presented by D. W. Palmer, www.semiconductors.co.uk, 2006.02 (unpublished). 